\title{
Predicted effects of pulse width programming in spinal cord stimulation: a mathematical modeling study
}

\author{
Dongchul Lee $\cdot$ Brad Hershey $\cdot$ Kerry Bradley $•$ \\ Thomas Yearwood
}

Received: 2 September 2009/Accepted: 6 April 2011/Published online: 29 April 2011

(C) The Author(s) 2011. This article is published with open access at Springerlink.com

\begin{abstract}
To understand the theoretical effects of pulse width (PW) programming in spinal cord stimulation (SCS), we implemented a mathematical model of electrical fields and neural activation in SCS to gain insight into the effects of PW programming. The computational model was composed of a finite element model for structure and electrical properties, coupled with a nonlinear double-cable axon model to predict nerve excitation for different myelinated fiber sizes. Mathematical modeling suggested that mediolateral lead position may affect chronaxie and rheobase values, as well as predict greater activation of medial dorsal column fibers with increased PW. These modeling results were validated by a companion clinical study. Thus, variable PW programming in SCS appears to have theoretical value, demonstrated by the ability to increase and even 'steer' spatial selectivity of dorsal column fiber recruitment. It is concluded that the computational SCS model is a valuable tool to understand basic mechanisms of nerve fiber excitation modulated by stimulation parameters such as PW and electric fields.
\end{abstract}

Keywords Spinal cord stimulation - Pulse width . Paresthesia - Dermatome - Implantable pulse generator . Neurostimulation $\cdot$ Mathematical nerve model $\cdot$ Non-linear nerve model

D. Lee $(\square) \cdot$ B. Hershey $\cdot$ K. Bradley

Boston Scientific Neuromodulation, 25155 Rye Canyon Blvd,

Valencia, CA 91355, USA

e-mail: Dongchul.Lee@bsci.com

T. Yearwood

Comprehensive Pain \& Rehabilitation, 28260 Highway 98,

Daphne, AL 36526, USA

\section{Introduction}

In 1965, Melzack and Wall proposed the gate control theory of pain, which paved the way for Shealy et al. to introduce spinal cord stimulation (SCS) as a treatment for chronic neuropathic pain in 1967 [1,2]. Over the past four decades, improvements have been made in both the clinical and technical aspects of SCS, including patient screening and follow-ups, and equipment design and functionality [3]. Some published reports suggested that there was therapeutic value in having a stimulation pulse width (PW) of long duration. Longer PWs have been anecdotally described as achieving better pain-paresthesia overlap and comfort for the patient, thus potentially more effective at relieving pain [4]. Gould and Bradley reported in a retrospective analysis of patient-preferred programs that over $50 \%$ of the programs used PWs in excess of $450 \mu$ s [9]. Recently, Yearwood et al. showed that 10/19 patients had greater coverage, and 8/19 patients displayed 'caudal shift' of paresthesia coverage with increased PW [5].

Computational modeling of neurons has been used to understand basic mechanisms for neural stimulation since Hodgkin and Huxley's mathematical model was described [6]. Neural activity is modulated by electric fields applied as intracellular current injection and external field stimulation. Ranck qualitatively discussed how changes in the extracellular voltage in the vicinity of an axon cause some regions of the axon membrane to depolarize and others to hyperpolarize [7].

An SCS computational model was initially developed by Coburn [8] and was significantly furthered by Holsheimer and colleagues, beginning with Struijk and Holsheimer's development of a three-dimensional field model of SCS [9]. The University of Twente (UT) model has been instrumental in pioneering advances in SCS [4]. Their 
Fig. 1 Depiction of the mesh of the FEM for the spinal cord and multicontact lead.

a Components and structure of model. b Model mesh —only the high density part (middle block) is shown. The mesh was segmented into sections of variable node density: near the contacts $(\leq 300 \mu \mathrm{m})$; insulator, dura, and spinal cord

$(\leq 750 \mu \mathrm{m})$; epidural space $(\leq 3000 \mu \mathrm{m})$; and

vertebral bone $(\leq 5000 \mu \mathrm{m})$
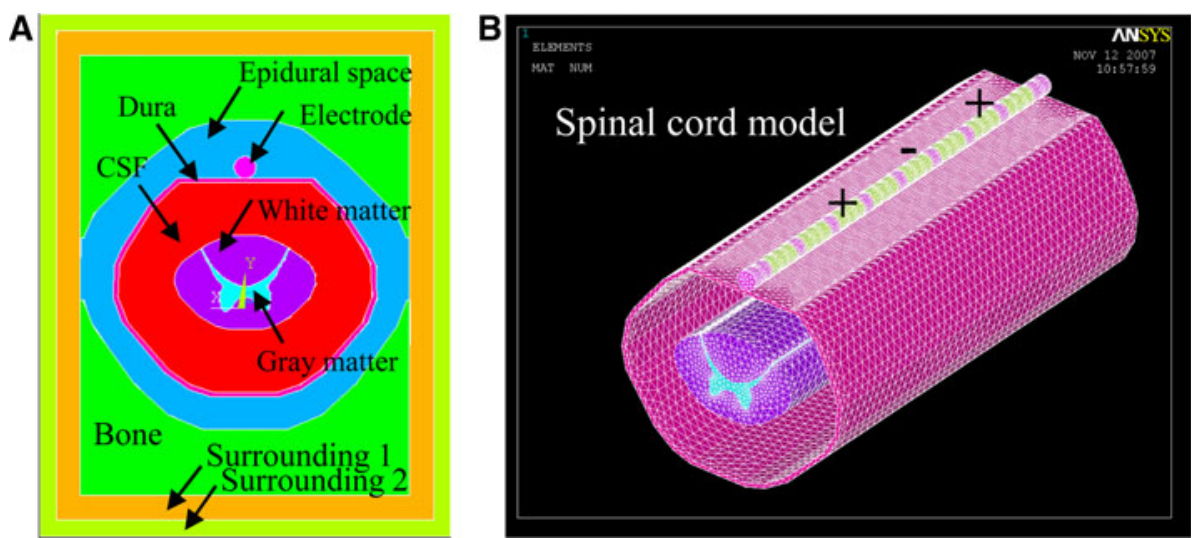

computational model estimated the effect of anatomic parameters on the thresholds of dorsal column (DC) fibers [10], predicted the potential location of excitation in dorsal root (DR) fibers [11], and analyzed the effect of cerebrospinal fluid (CSF) thickness [12]; with clinical validation of most of the conclusions from the modeling studies [13-15]. The model contributed significantly to the specification of contact design parameters such as size and spacing [16, 17], to favor preferential stimulation of DC fibers over root fibers [18].

The effects of PW, however, had not been evaluated in a mathematical modeling study. To investigate these effects, we generated a model of SCS (Boston Scientific Neuromodulation (BSN) model) based generally on the approach of the UT low-thoracic model [4, 19], but with several myelinated fiber sizes distributed within the DC based on histological data $[11,20,21]$. The incorporation of multiple fiber sizes was felt to be critical, because the effects of PW are known to be fiber size dependent [22]. The BSN model also used previously-developed non-linear myelinated mammalian fiber models [23].

\section{Objectives}

Using the BSN mathematical model, our objective was to better understand the effect of PW programming in SCS upon technical results: strength-duration threshold parameters, fiber recruitment, and loci of activated fiber regions.

\section{Methods}

\subsection{Mathematical model}

A new three dimensional finite element mathematical (FEM) model was created of the low-thoracic spinal cord and its surrounding environment. The FEM model consisted of spinal cord white and gray matter, cerebrospinal fluid, dura, epidural space tissue, vertebral bone, and a cylindrical multicontact lead. Spinal cord (white and gray matter boundary) was modeled on the shape/size of the cord at the low-thoracic vertebral level (using images from human cadaver samples) [21]. Dura cross section and thickness $(270 \mu \mathrm{m})$ was approximated from a recent model of motor cortex stimulation [24]. The inner portion of the vertebral bone was round in shape, but its outer surface was square/flat for computational simplicity. The surrounding layer 2 (Fig. 1) was treated as ground, while surrounding layer 1 was used to adjust electrode impedance between contact and the ground layer. Once cross sections of necessary compartments (white and gray matter, dura etc.) were identified, each compartment was extruded into three blocks in the rostro-caudal direction. Each block has a rostrocaudal length of 70,35 , and $70 \mathrm{~mm}$. Therefore, total rostrocaudal length of the model is $175 \mathrm{~mm}$ which is long enough to minimize the boundary effect on potential distribution. The model of the stimulating lead is placed in the middle block, which has a length of $35 \mathrm{~mm}$ and has

Table 1 Resistivity values and density of nodes of the FEM domains $[4,25]$

\begin{tabular}{lll}
\hline Material & $\begin{array}{l}\text { Resistivity } \\
(\Omega \mathrm{mm})\end{array}$ & $\begin{array}{l}\text { Density (nodes/ } \\
\left.\mathrm{mm}^{2}\right)\end{array}$ \\
\hline White matter (longitudinal) & 1666 & 150 \\
White matter (transverse) & 12,048 & \\
Gray matter & 4347 & 101 \\
$\mathrm{CSF}$ & 588 & 80 \\
Epidural space & 25,000 & 39 \\
Dura & 1666 & 113 \\
Vertebral bone & 50,000 & 0.6 \\
Electrode contact & 0.0002 & 16,588 \\
Electrode insulator & $10^{9}$ & \\
Surrounding layer 1 & 250,000 & 0.5 \\
Surrounding layer 2 & 0.0002 & \\
\hline
\end{tabular}


significantly higher density (Table 1) of nodes compared to the first and last block.

The lead consisted of eight cylindrical platinum-iridium contacts (conducting domains were $3 \mathrm{~mm}$ length and $1.25 \mathrm{~mm}$ diameter), separated by $1 \mathrm{~mm}$ lengths of insulating polymer (non-conducting domains of $1 \mathrm{~mm}$ length). The lead was positioned dorsally, atop the dura and aligned with the midline of the spinal cord. The geometry of the model is illustrated in Fig. 1a and electrical resistivities and node density are given in Table 1, values coming predominantly from the literature $[4,25]$. The volume was meshed with over 1 million nodes with tetrahedron shaped elements, with a high-density mesh in the region close to where electrodes are located as illustrated in Fig. $1 \mathrm{~b}$.

The spinal cord geometry (Fig. 2) was created using a combination of features from relevant literature sources. The cross-section of the cord was derived from Kameyama et al., and the DR trajectory of Struijk et al. was adopted $[11,21]$. Each DR was modeled as a larger diameter 'mother' fiber connected to bifurcated 'daughter' fibers of smaller diameter (Fig. 2b).

The electric potential was computed by solving the model using the ANSYS (Canonsburg, PA) Emag analysis tool, employing the iterative equation solver with Jacobi Conjugate Gradient method. A unit current was injected at the center point of any active contact with current return to ground (surrounding layer 2). To compute the electric potential generated by multiple contacts, multiple simulations with current injection from each individual contact alone were performed. The solutions were then superimposed to generate the net electric potential in the spinal cord (white and gray matter). Finally, the solution was extracted and post-processed in Matlab (Natick, MA).

\subsection{Double cable axon model}

The double cable axon model was developed by McIntyre et al. based on mammalian motor nerve fibers with geometrically and electrically accurate parameters (http://senselab. med.yale.edu/) implemented in NEURON [23, 26]. The model incorporated explicit representations of the nodes of Ranvier, paranodal and internodal sections of the axon as well as a finite impedance myelin sheath. This model matched experimental data by combining an accurate representation of the ion channel and the geometry of the paranode, internode, and myelin. The model has nonlinear membrane dynamics of ion channels at the nodes of Ranvier. The ion channel includes fast $\mathrm{Na}+$, persistent $\mathrm{Na}+$, and slow $\mathrm{K}+$ conductances. The node of Ranvier contains these ion channels in addition to a linear leakage conductance, and the membrane capacitance.

\subsection{Simulation procedure}

For the various model investigations, the voltage data from the FEM model was ported to Matlab for interpolation on regular grid points in 3D space. DC fibers were placed on regular grid $(200 \mu \mathrm{m}$ for mediolateral direction and $100 \mu \mathrm{m}$ for dorsoventral direction; see Fig. 2a) and projected in the rostrocaudal direction. The interpolated voltage along the corresponding fiber (Fig. 2c) was applied to non-linear axon models [23] with different fiber diameters for DC and DR fibers using the extracellular mechanism capability in NEURON. To apply a guarded cathode (anode-cathode-anode, where each anode provides half of the total anode current; a commonly programmed contact combination in SCS), the electric potential generated from the three contacts individually was summed based on superposition principle of current sources in space. Fiber
Fig. 2 Structure of spinal cord model. a Transverse view of spinal cord and location of dorsal column fibers. b Dorsal roots are composed of a mother fiber and bifurcated daughter fibers. The trajectory of the mother fiber was digitized from Struijk 1993. c Threedimensional view of spinal cord and DR fibers
A

Transversal view of spinal cord model

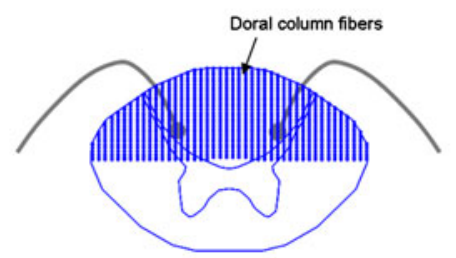

B

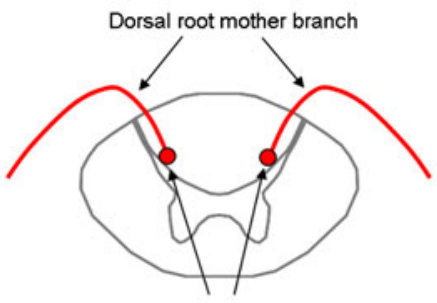

Dorsal root daughter branch

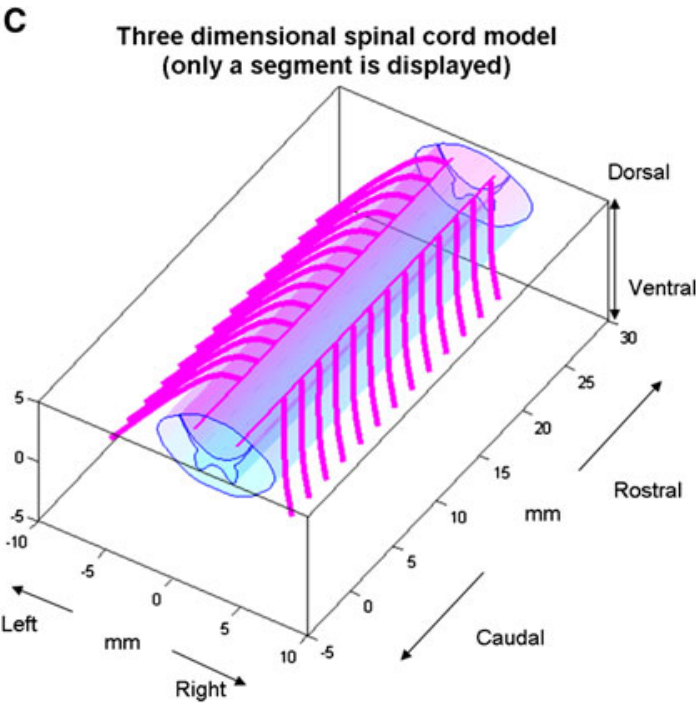


activation was defined when an action potential was observed at a minimum of five nodes for a depolarized fiber. During simulation, anodic break did not occur because the anode intensity was relatively weaker than cathode, and the axon's ion channel dynamic was not sensitive to anodic break.

\subsection{Fiber distribution}

The new FEM SCS model includes several myelinated fiber sizes (5.7-14 $\mu \mathrm{m}$ diameter) for both $\mathrm{DC}$ and DR fibers. In the human DC, the density and distribution of various fiber sizes changes from the midline to the DR entry zone, with medium and larger sized fibers trending toward greater density in the lateral DCs [20] (see Fig. 3a, b). Thus, the distribution of DC fibers in the model was designed to reflect these variations.

The number of stimulated fibers for specific diameter and location was estimated from the density (Fig. 3b) assuming that the characteristic of mediolateral distribution is preserved dorsoventrally.

\subsection{Model investigations}

To study the effect of PW on DC fibers, a longitudinal guarded cathode combination (anode-cathode-anode with separations of $8 \mathrm{~mm}$ center-to-center) parallel to DC fibers was applied to the model with different PWs $(60,210,450$, and $1000 \mu \mathrm{s})$ monophasic square pulse. The centerto-center contact separation of the modeled lead is $4 \mathrm{~mm}$, which matches commercially available SCS percutaneous leads (Boston Scientific Linear ST). The $8 \mathrm{~mm}$ separation between anode and cathode is selected because it reflects commonly-programmed combinations. Although commercial current-controlled stimulators use square pulses, followed by an interphase interval (typical: $100 \mu \mathrm{s}$ ), and a low-voltage passive recharge interval (typical: $6 \mathrm{~ms}$ ), our simulation ignored the effect of recharge phase because a $100 \mu$ s interval after the stimulation phase is long enough to eliminate any impact of recharge phase [27]. Selection of PWs was intended to match the breadth of programmable values in commercial devices.

The thickness of the CSF was set at $3.2 \mathrm{~mm}$ [15]. The upper limit of stimulation current was defined using both the threshold of the most excitable DR fiber (DRth) fiber (15 $\mu \mathrm{m}$ diameter), and 1.4 times the threshold of the firstactivated $11.5 \mu \mathrm{m}$ DC fiber $(1.4 *$ Pth), which is believed to generate initial paresthesia during SCS [10]. The rationale for having both limits was the clinical observation that some patients report that their maximum comfortable level of stimulation does not activate fibers believed to be DR fibers (i.e., they do not report abdominal cramping); in these cases, the stimulation is perceived as 'too intense' in caudal regions and the patient becomes uncomfortable [28].

\subsection{Clinical investigation}

A companion clinical study into the technical outcomes of PW programming (paresthesia thresholds and body coverage) in SCS was also performed [5, 29]. Briefly, subjects utilizing fully-implanted Precision ${ }^{\mathrm{TM}}$ SCS systems for chronic intractable pain were enrolled. Clinical data was obtained from an IRB-approved post-market clinical investigation of $\mathrm{PW}$ programming in spinal cord stimulators (ClinicalTrials.gov \# NCT00399516). All subjects had percutaneous leads positioned in the dorsal epidural space at the T7-T9 vertebral level to treat low back and lower extremity pain with the Boston Scientific Precision SCS device and were screened for inclusion and exclusion criteria [5]. Patients who met the necessary criteria underwent the informed written consent process per the Declaration of Helsinki. Table 2 summarizes the gender, age, diagnosis, location of pain, and time-since-implant of the subjects.

In the study, the favorite program of each subject was used, with only the PW parameter varied from 50 to $1000 \mu \mathrm{s}$, in a pre-randomized order. At each PW, the pulse amplitude was increased from zero, first to perception
Fig. 3 a Cross section of spinal cord with grid overlay to quantify the fiber diameter distribution $\mathbf{b}$ as a function of distance from midline, parameterized by fiber size [20]

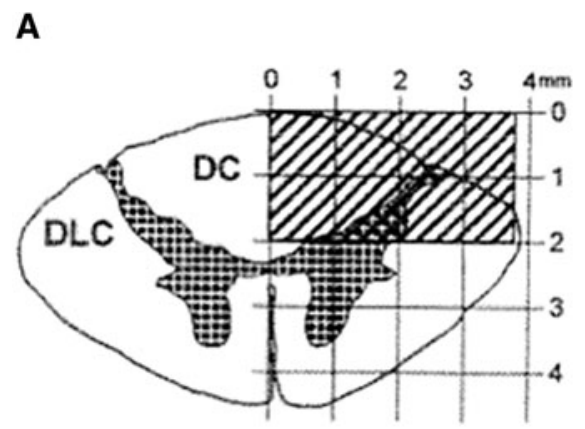

Feirabend et al. Brain 2002

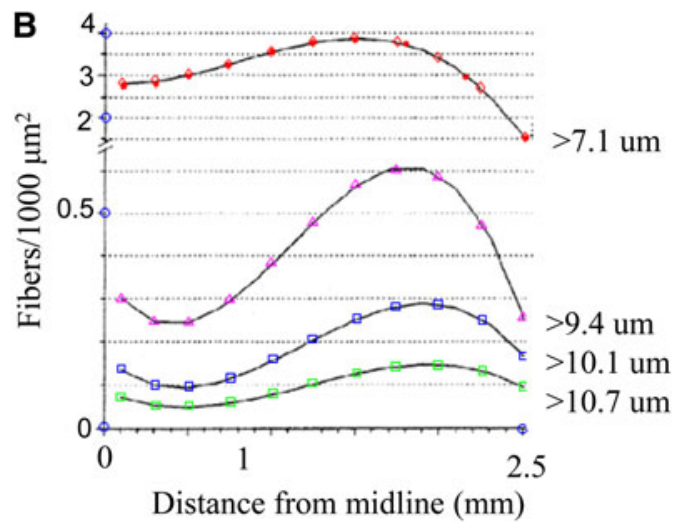


Table 2 Demographic statistics of enrolled subjects

\begin{tabular}{ll}
\hline Number tested & 19 \\
Gender & $11 \mathrm{M} / 8 \mathrm{~F}$ \\
Age & 53 (46-57) years \\
$\begin{array}{l}\text { Diagnoses (in order of } \\
\text { prevalence among enrolled } \\
\text { subjects) }\end{array}$ & $\begin{array}{c}\text { Neuropathy/radiculopathy, post- } \\
\text { surgical neuropathy } \\
\text { (laminectomy, decompression), } \\
\text { Pain location }\end{array}$ \\
& CRPS \\
Time since implant & Primary bilateral lower extremity, \\
& $2.3(1.3-5.1)$ months \\
\hline
\end{tabular}

threshold, which was recorded, then to a strong-but-comfortable level, at which point the subject was asked to draw the extent of their paresthesia over a displayed human figure on a pen tablet PC. Analyses of the perception thresholds were performed to create strength-duration curves from which chronaxie and rheobase estimates were made. Also, the paresthesia drawings were analyzed to look for changes in total body coverage, as well as shifts in the location of the paresthesia on the body as they related to PW. A total of 19 subjects completed testing and the clinical results were published [29].

\subsection{Data analysis}

In SCS programming, a common clinical observation is that the paresthesia 'spreads' to multiple dermatomes as the pulse amplitude is increased [30]. In previous mathematical modeling studies of SCS, increased stimulation amplitude increased the calculated cross-sectional area of stimulated DC fibers [4]. The combination of these phenomena suggests that, as amplitude is increased, a greater depth and breadth of DC fiber activation is responsible for the increased spread of paresthesia. Based on this relationship, the cross-sectional area of stimulated DC fibers was used to estimate 'relative' paresthesia coverage change in the computational model to study the effect of PW.

Anatomic studies of the DC have suggested a spatiotopic mapping of dermatomes, where lateral DC fibers represent relatively rostral dermatomes, and medial DC fibers are projections of DR fibers from more caudal dermatomes [31]. Although the boundary of each dermatome in the DC is not clearly divided, we assumed that the gross characterization of rostral versus caudal dermatomes could be assessed in the model. Therefore, for all fibers recruited by stimulation, medial fibers (MedF) were defined as those located within $600 \mu \mathrm{m}$ of the spinal cord midline, and lateral fibers (LatF) as those located greater than $600 \mu \mathrm{m}$ from the midline [32]. Using these definitions, the location of the focus of paresthesia for each PW was estimated by computing the total number of stimulated fibers from the
Fiber diameters

(population in total column fibers)

14.0 um $(0.025 \%) \quad 12.8$ um $(0.08 \%)$

11.5 um $(0.25 \%) \quad 8.7$ um (3\%)

$7.5 \operatorname{um}(10 \%) \quad 5.7$ um (25\%)

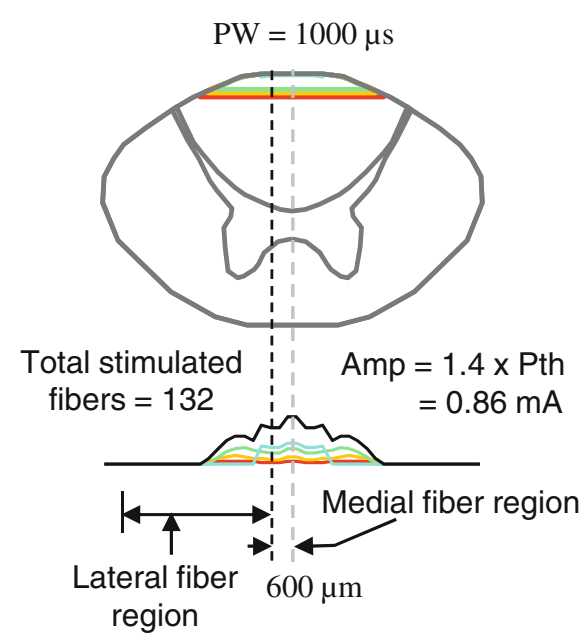

LatF: Stimulated fibers from lateral fiber region
MedF: Stimulated fibers from medial fibers region

Fig. 4 Analysis method to compute the number of stimulated fiber from lateral versus medial regions. For all fibers recruited by stimulation, medial fibers (MedF) were defined as stimulated fibers located within $600 \mu \mathrm{m}$ of the spinal cord midline, and lateral fibers (LatF) as stimulated fibers located greater than $600 \mu \mathrm{m}$ from the midline [32]. Using these definitions, the location of the focus of paresthesia for each PW was estimated by computing the total number of stimulated fibers from the medial (MedF) and lateral (LatF) regions and their ratio (LatF/MedF). Legend shows fiber diameter and corresponding colors. Percentage represents population of the corresponding size fibers out of total population in dorsal column fibers [20]

medial $(\mathrm{MedF})$ and lateral (LatF) regions and their ratio (LatF/MedF) (Fig. 4).

\section{Results}

The effects of varying PW on DC fiber recruitment were studied using the finite-element model. To quantify the recruited region in the DCs, two metrics were calculated: (1) cross-sectional area of stimulated fibers in a transverse slice of the DCs underneath the cathode; (2) the ratio between LatF and MedF was computed and plotted versus PW.

\subsection{Strength-duration curves}

For a given PW, stimulation amplitudes of DCth and DRth were applied. As expected, a classic strength-duration relationship was observed, where the pulse amplitude decreased 


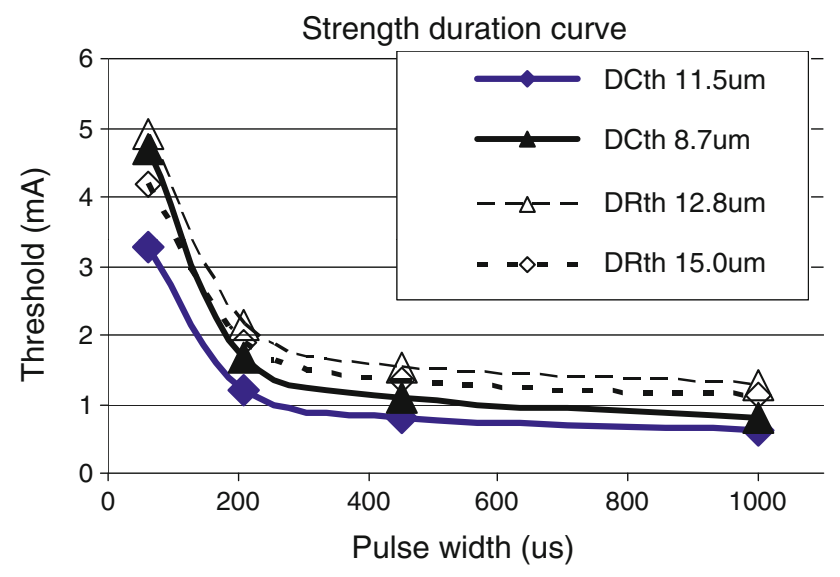

Fig. 5 Strength-duration curve of DC (11.5 and $8.7 \mu \mathrm{m}$ diameter) and DR (12.8 and $15.0 \mu \mathrm{m}$ diameter) fibers. Depending on electrode placement, nerve fibers stimulated at perception threshold might be different and might have different values of chronaxie and rheobase. Fiber sizes were selected to show that the strength-duration curve with different PWs can depend upon fiber diameter

nonlinearly as a function of increasing PW. Chronaxie and rheobase were $285 \mu \mathrm{s}(266-303)$ and $0.58 \mathrm{~mA}(0.5-0.7)$, respectively [33]. A main source of variability was lead position, where more lateral positions $(0.25-1.8 \mathrm{~mm}$ from midline) resulted in lower chronaxie and rheobase. Further, the model predicted that insufficient data (e.g., using a PW range up to only $1000 \mu \mathrm{s}$ ) results in errors of $\sim 22 \%$ overestimation for chronaxie [33].

The strength-duration curve in Fig. 5 shows that short PWs (e.g., $60 \mu \mathrm{s})$ may stimulate DR fibers $(15.0 \mu \mathrm{m})$ before DC fibers $(8.7 \mu \mathrm{m})$. However, this recruitment order is reversed as PW increased, suggesting that wider
PW values may preferentially recruit DC fibers over DR fibers.

\subsection{Stimulated DC fibers with varied PW}

To assess the effect of PW on the recruitment of different fiber diameters, recruitment contours in the DC were plotted by fiber size (Fig. 6). From these contours, a spatial histogram of the total number of fibers recruited was created. Figure 6 shows that, in addition to more total fibers being recruited at larger PW, the mediolateral distribution of the recruitment was changed by increasing PW. For short PW $(60 \mu \mathrm{s})$, the mediolateral distribution is flat, but as the PW increased, more medial fibers were recruited. In addition, the cross-sectional area of all DC fiber diameters was increased for both amplitudes (1.4Pth and DRth).

Interestingly, for a PW of $60 \mu$ s at $1.4 *$ Pth, some DR fibers were stimulated (data not shown). However, for all PW $>60 \mu \mathrm{s}$, no DR fibers were stimulated at $1.4 *$ Pth amplitude.

\subsection{Cross-sectional DC area versus PW}

The recruited cross-sectional DC area of each fiber diameter for different PWs is shown in Fig. 7. We compared the model results in Fig. $7 \mathrm{~b}$ to results from the aforementioned companion clinical study where paresthesia coverage changes with increasing PW were measured and are plotted in Fig. 7a [5]. There appeared to be reasonable agreement between the modeling and clinical results, as the observed trend of increase was clearly shown [5, 34] over the entire PW range. Specifically, paresthesia coverage increased
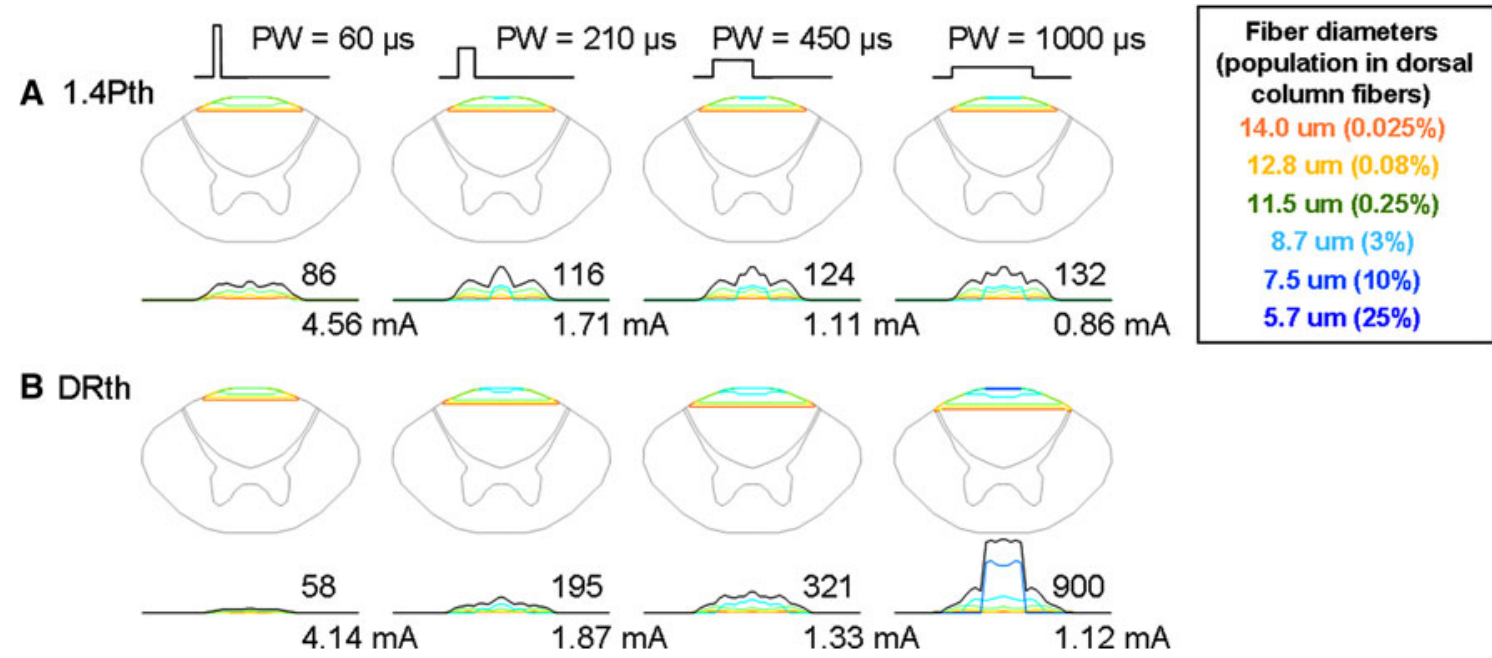

Fig. 6 Stimulated DC fibers segmented by fiber diameter for varied PW and two pulse amplitude intensities (1.4* Pth, DRth). Along the top of the figure is a graphic reflecting the width and amplitude of the applied stimulation pulse. Below this are the model-generated recruitment contours shown in a transverse slice of the spinal cord.
The model contours in each spinal cord slice are segmented by fiber diameter, as shown in the Legend. Below each spinal cord slice is the mediolateral histogram of total number of fibers stimulated for each stimulation pulse 

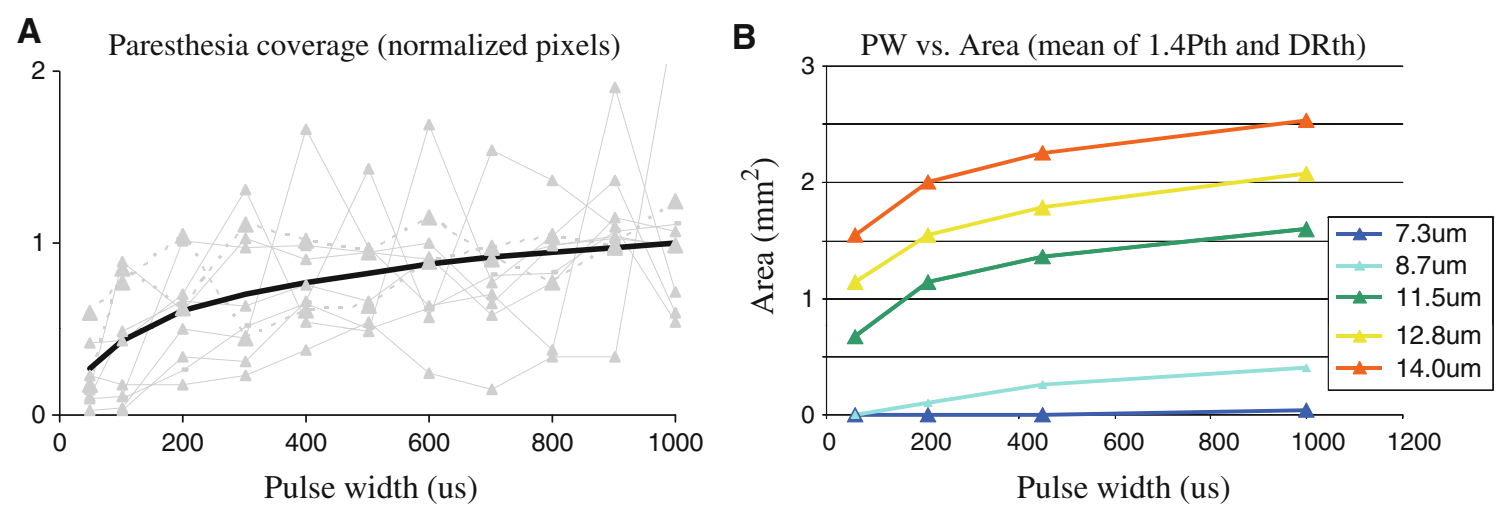

Fig. 7 Effect of PW on paresthesia coverage. a Normalized total paresthesia coverage from 19 patients as a function of pulse width [5]. b Crosssectional recruited area for each fiber type with different PW. Results are averages of the two stimulation conditions (1.4Pth and DRth)

greatly in the lower range of PW values (60-300 $\mu \mathrm{s})$ and more gradually at PW values above $500 \mu$ s.

In our computer model, the cross-sectional area of DC fibers was computed at different PW values, increasing from 60 to $1000 \mu \mathrm{s}$ (Fig. 7b). The area of recruitment within the range of PW of 60 to $200 \mu$ s was faster than that of the upper range of PW ( $>450 \mu \mathrm{s})$, which is consistent with the observed clinical trend (Fig. 7a). The smallest DC fibers $(<9.0 \mu \mathrm{m}$ diameter) were rarely recruited at short PW and the recruitment increased significantly at moderate-to-long PW values $(>400 \mu \mathrm{s})$. Because of the relative abundance of small fibers, however, the number of recruited small fibers overwhelmed large fibers (Fig. 6).

In summary, the computational model showed that the cross-sectional area of DC fibers increased by widening the
PW, which appears to match recent clinical observation. Longer PW was more effective in stimulating smaller diameter fibers than shorter PW, and increased total stimulated fibers, presumably because of the abundance of small fibers located within the superficial and medial dorsal columns.

\subsection{Fiber ratio versus $\mathrm{PW}$}

The ratio of lateral-to-medial fibers recruited was calculated for each PW used in the finite-element model. Under the assumption that lateral fibers corresponded to more rostral dermatomes and medial fibers originated from more caudal dermatomes, this ratio attempts to quantify the degree of expected caudal dermatomal shift in the paresthesia
Fig. 8 Fiber ratio (LatF/MedF) from FEM model was compared to regression line from 6 patient 'responders' [5, 34]. Increasing $\mathrm{PW}$ in the model resulted in more medial than lateral fiber recruitment, which might be related to the clinical observation of increasing caudal dermatome coverage with larger PW (via lower-lumbar and sacral fiber recruitment located in medial portions of DC)

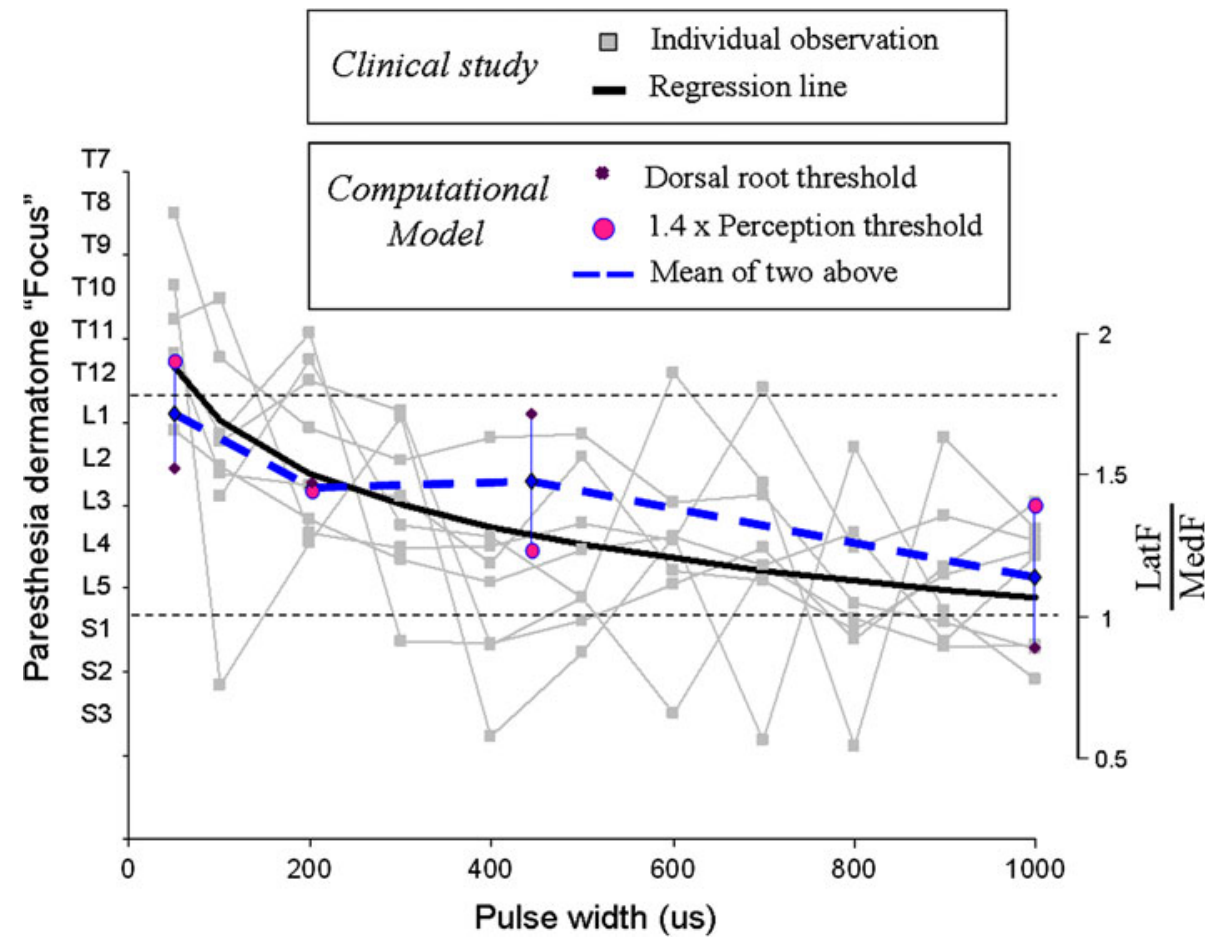


observed clinically. As shown in Fig. 8, the fiber ratio decreased as the applied PW was increased, thereby predicting that wider PW would recruit more medial fibers than shorter PW. Figure 8 shows this fiber ratio superimposed upon the data from the companion clinical study [5, 34]; this data is from subjects who demonstrated a significant shift in the median dermatomal location of their total paresthesia with increasing PW, i.e., a 'sacral shift.' Good agreement is seen between the FEM model prediction and the clinical data from patient 'responders to sacral shift'.

\section{Discussion}

In neurostimulation applications, the pulse amplitude and width contribute directly to depolarizing the cell membrane and are thus critical parameters for determining the locus of excited tissue. The clinical effect of PW on paresthesia coverage and location was recently reported [5]. Our computational model was used to understand the possible mechanisms responsible for observed paresthesia distribution change with $\mathrm{PW}$.

\subsection{Fiber recruitment changes with PW}

This study was the first to implement realistic fiber diameter and spatial distributions (based upon histologic studies of DC fibers in human cadavers) in a computational spinal cord model. Although there are several limitations (see below) in the model, the realistic fiber distribution was a critical component in suggesting a reason for the clinicallyobserved paresthesia coverage increases and 'sacral shift' of paresthesia with increased PW. The computational model showed that the cross-sectional area of recruited DC fibers increased by widening PW. The trend of recruited DC area increase as a function of PW was consistent with clinical observations [5]. In addition, the fiber ratio between fibers from medial and lateral sections of the DC reproduced the clinically-observed trend of 'sacral shift' for PW increase, assuming that medial DC fibers innervated more caudal dermatomes as compared to lateral DC fibers. Finally, the strength-duration curve from DC and DR fibers had different characteristics, which corresponds to the observation that some subjects in the companion clinical study had reduced abdomen paresthesia with higher PW [29]. Therefore, the computational model reflected two major effects of $\mathrm{PW}$ on paresthesia: total coverage and paresthesia location.

The computational model was not able to explain pure paresthesia shifts; certain body areas covered by paresthesia at short PW were no longer reported to have paresthesia at long PW. This phenomenon may result from a change in neural recruitment, or it could be due to attentional shift. For example, if the paresthesia intensity is strong in a new body area, the subject may have difficulty focusing on the perception of paresthesia in other body areas; if the leg paresthesia becomes strong as a result of more DC fiber recruitment, the relatively weaker abdomen paresthesia resulting from DR stimulation may be less noticeable. Such attentional shift might be used to maximize the target coverage by increasing contrast between targets versus side-effect stimulation.

\subsection{Limitations}

The fiber diameter distribution was derived from the superficial layer $(<500 \mu \mathrm{m})$ of the dorsal columns [20]. There is no information how this distribution might change for deeper fibers. In this study, it was assumed that the distribution would be consistent in the dorsoventral direction, and if this assumption proved to be false, some of the reported results may be affected.

The effect of DR fibers was not included. As mentioned previously, at $60 \mu$ s with $1.4 *$ Pth amplitude, DR fibers close to the cathode were stimulated. Since the finite-element model assumes a mid- to low-thoracic lead placement, such DR fiber recruitment would clinically manifest as paresthesia in thoracic dermatomes (e.g., abdomen, ribs, midback). Such paresthesias were observed clinically in several patients at lower PW settings. Thus, it is likely that the model results understate the degree of mediolateral shift in DC recruitment by not including DR effects.

The tissue conductivities used in the FEM were implemented from published papers [12, 14, 35], but epidural space conductivity was tuned to match the monopolar impedance of percutaneous leads $(370 \Omega$ ) to the clinicallyobserved average value $(368 \pm 98 \Omega)$ on chronicallyimplanted leads [36]. Epidural space (fat) conductivity plays an important role in determining the direction and magnitude of the current flow (e.g., ventrally through dura versus dorsolaterally and/or dorsally in the epidural space). Thus, the quantitative results from the finite-element model might be related to the conductivity of the epidural space. Similarly, the conductivity of other tissues local to the stimulating contacts (e.g., dura mater) may affect the results (computational and clinical).

The dorsal column axons and nodes of Ranvier were based upon a popular non-linear dynamic neuron model derived from motor axons [37-39]. Thus, it may have limited capability in representing the behavior of sensory nerves.

The boundary line between medial and lateral fibers was selected arbitrarily at $600 \mu \mathrm{m}$ from the spinal cord physiological midline. Also, the number of recruited lateral and medial fibers is highly dependent on the fiber distribution 
of each fiber size. While the trends of mediolateral recruitment with increased PW would be maintained, it is expected that different results for the change in fiber ratio with PW would be found if different fiber distributions and the lateral-medial fiber boundary were used.

Maximum comfortable thresholds in mathematical model were provided from two clinical observations: discomfort was reported by patients at levels approximately 1.4 times the perception threshold $(1.4 * \mathrm{Pth})$, and DR stimulation at a T7-T9 vertebral level is often poorly tolerated (DRth) [13]. These assumptions have several challenges. First, it is difficult to prove that $1.4 *$ Pth in clinical application is the same as $1.4 *$ Pth in a computer model. Second, the actual source of discomfort is not known. At maximum stimulation amplitude, patients may complain of 'loss of limb control' and 'strong vibration in limb' without any obvious evidence of dorsal root stimulation. Therefore, results may change quantitatively by defining the maximum comfortable amplitude.

Fibrous encapsulation of electrodes is known to degrade stimulation electric fields by (1) further separating electrode from target axons and (2) introducing a low resistivity "shunt path" layer of saline between the lead and the fibrous encapsulation which may "stretch" the electric field parallel to the lead axis. However, the general principle to recruit small medial fibers would hold for large PW stimulation.

\section{Conclusions}

Predictions of the effect of PW in SCS with a mathematical model incorporating realistic fiber size, density, and spatial distributions, include: (A) a classic strength-duration relationship exists for DC fiber recruitment, where lead position may affect the chronaxie; (B) DC area is increased with larger PW values; and (C) larger PWs increase the relative number of medial DC fibers recruited, relative to lateral DC fibers.

Our modeling predictions showed good agreement with clinical data such as increased paresthesia coverage (increased cross section area of DC fibers) and sacral shift (decreased LatF/MedF for larger PW). We note that the inclusion of the fiber diameter distribution into the DC's in our model was critical to providing insight into the clinically-observed 'sacral shift' paresthesia phenomenon.

Acknowledgments This study was sponsored by Boston Scientific Neuromodulation. Dr. Thomas Yearwood is an independent consultant for Boston Scientific Neuromodulation, and recipient of research grant monies used to fund this research project. Brad Hershey, Dongchul Lee, and Kerry Bradley are employees of Boston Scientific Neuromodulation.
Open Access This article is distributed under the terms of the Creative Commons Attribution Noncommercial License which permits any noncommercial use, distribution, and reproduction in any medium, provided the original author(s) and source are credited.

\section{References}

1. Melzack R, Wall PD (1965) Pain mechanisms: a new theory. Science 150:971-979

2. Shealy CN, Mortimer JT, Reswick JB (1967) Electrical inhibition of pain by stimulation of the dorsal columns: preliminary clinical report. Anesth Analg 46:489-491

3. Barolat G (1995) Current status of epidural spinal cord stimulation. Neurosurg Q 5:98-124

4. Holsheimer J (2002) Which neuronal elements are activated directly by spinal cord stimulation. Neuromodulation 5:25-31

5. Yearwood TL, Hershey B, Bradley K, Lee D (2010) Pulse width programming in spinal cord stimulation: a clinical study. Pain Physician 13:321-335

6. Hodgkin AL, Huxley AF (1952) A quantitative description of membrane current and its application to conduction and excitation in nerve. J Physiol 117:500-544

7. Ranck JB Jr (1975) Which elements are excited in electrical stimulation of mammalian central nervous system: a review. Brain Res 98:417-440

8. Coburn B (1980) Electrical stimulation of the spinal cord: twodimensional finite element analysis with particular reference to epidural electrodes. Med Biol Eng Comput 18:573-584

9. Holsheimer J, Struijk JJ (1988) Analysis of spinal cord stimulation. In: Wallinga W, Boom HB, De Vries J (eds) Electrophysiological kinesiology, vol 804. Excerpta Medica Congress Series, Amsterdam, pp 95-98

10. Struijk JJ, Holsheimer J, van der Heide GG, Boom HB (1992) Recruitment of dorsal column fibers in spinal cord stimulation: influence of collateral branching. IEEE Trans Biomed Eng 39:903-912

11. Struijk JJ, Holsheimer J, Boom HB (1993) Excitation of dorsal root fibers in spinal cord stimulation: a theoretical study. IEEE Trans Biomed Eng 40:632-639

12. Struijk JJ, Holsheimer J, Barolat G, He J, Boom HB (1993) Paresthesia thresholds in spinal cord stimulation: a comparison of theoretical results with clinical data. IEEE Trans Rehabil Eng 1:101-107

13. He J, Barolat G, Holsheimer J, Struijk JJ (1994) Perception threshold and electrode position for spinal cord stimulation. Pain 59:55-63

14. Holsheimer J, Barolat G, Struijk JJ, He J (1995) Significance of the spinal cord position in spinal cord stimulation. Acta Neurochir Suppl 64:119-124

15. Holsheimer J, den Boer JA, Struijk JJ, Rozeboom AR (1994) MR assessment of the normal position of the spinal cord in the spinal canal. AJNR Am J Neuroradiol 15:951-959

16. Holsheimer J, Struijk JJ (1992) Electrode geometry and preferential stimulation of spinal nerve fibers having different orientations: a modeling study. In: 14th Annual international conference of the IEEE engineering in medicine and biology society, Paris, France, pp 1374-1375

17. Holsheimer J, Wesselink WA (1997) Optimum electrode geometry for spinal cord stimulation: the narrow bipole and tripole. Med Biol Eng Comput 35:493-497

18. Holsheimer J, Struijk JJ, Tas NR (1995) Effects of electrode geometry and combination on nerve fibre selectivity in spinal cord stimulation. Med Biol Eng Comput 33:676-682 
19. Holsheimer J, Barolat G (1998) Spinal geometry and paresthesia coverage in spinal cord stimulation. Neuromodulation 1: 129-136

20. Feirabend HK, Choufoer H, Ploeger S, Holsheimer J, van Gool JD (2002) Morphometry of human superficial dorsal and dorsolateral column fibres: significance to spinal cord stimulation. Brain 125:1137-1149

21. Kameyama T, Hashizume Y, Sobue G (1996) Morphologic features of the normal human cadaveric spinal cord. Spine 21: $1285-1290$

22. Burton C (1975) Dorsal column stimulation: optimization of application. Surg Neurol 4:171-179

23. McIntyre CC, Grill WM (2002) Extracellular stimulation of central neurons: influence of stimulus waveform and frequency on neuronal output. J Neurophysiol 88:1592-1604

24. Holsheimer J, Manola L (2004) Neuromodulation in epilepsy and chronic pain. Neuromodulation 9:143-153

25. Wesselink WA, Holsheimer J, King GW, Torgerson NA, Boom HBK (1999) Quantitative aspects of the clinical performance of transverse tripolar spinal cord stimulation. Neuromodulation 2:5-14

26. Hines ML, Carnevale NT (1997) The NEURON simulation environment. Neural Comput 9:1179-1209

27. Gorman PH, Mortimer JT (1983) The effect of stimulus parameters on the recruitment characteristics of direct nerve stimulation. IEEE Trans Biomed Eng 30:407-414

28. North RB, Fowler K, Nigrin DJ, Szymanski R (1992) Patientinteractive, computer-controlled neurological stimulation system: clinical efficacy in spinal cord stimulator adjustment. J Neurosurg 76:967-972

29. Yearwood T, Hershey B, Bradley K, Mekel-Borov N, Schnitzer J (2009) Pulse width and dorsal root stimulation in spinal cord stimulation. In: 13th North American neuromodulation, Las Vegas, NV

30. Hunter J, Ashby P (1984) Secondary changes in segmental neurons below a spinal cord lesion in man. Arch Phys Med Rehabil 65:702-705
31. Smith MC, Deacon P (1984) Topographical anatomy of the posterior columns of the spinal cord in man. The long ascending fibres. Brain 107(Pt 3):671-698

32. Lee DC, Hershey B, Bradley K, Moffitt M, Peterson D, Yearwood T (2007) Dorsal column selectivity in pulse width (PW) programming of spinal cord stimulation (SCS): computational model for the "sacral shift". In: 11th North American neuromodulation society Acapulco, Mexico

33. Yearwood T, Varga C, Hershey B, Lee DC, Moffitt M, Bradley K (2006) Strength-duration curves in spinal cord stimulation. In: 10th North American neuromodulation society, Las Vegas, NV

34. Yearwood T, Hershey B, Bradley K (2007) Dorsal column selectivity in pulse width (PW) programming of spinal cord stimulation (SCS): the "sacral shift". In: 11th North American neuromodulation society, Acapulco, Mexico,

35. Struijk JJ, Holsheimer J, van Veen BK, Boom HB (1991) Epidural spinal cord stimulation: calculation of field potentials with special reference to dorsal column nerve fibers. IEEE Trans Biomed Eng 38:104-110

36. Oakley JC, Prager J, Krames E, Weiner R, Stamatos J, Bradley K (2004) Variability of contact impedance over time in SCS. In: American Society stereotactic and functional neurosurgery biennial meeting, Cleveland, $\mathrm{OH}$

37. Butson CR, McIntyre CC (2007) Differences among implanted pulse generator waveforms cause variations in the neural response to deep brain stimulation. Clin Neurophysiol 118:1889-1894

38. McIntyre CC, Miocinovic S, Butson CR (2007) Computational analysis of deep brain stimulation. Expert Rev Med Devices 4:615-622

39. Miocinovic S, Parent M, Butson CR, Hahn PJ, Russo GS, Vitek JL, McIntyre CC (2006) Computational analysis of subthalamic nucleus and lenticular fasciculus activation during therapeutic deep brain stimulation. J Neurophysiol 96:1569-1580 